\title{
Withdrawal symptoms in long term sedation exposure of pediatric intensive care patients
}

\author{
Susanne Kotz \\ University Medical Center Hamburg-Eppendorf, Germany \\ Correspondence: Susanne Kotz. Address: Stueckenstraße 59, 22081 Hamburg, Germany. Telephone: \\ 49-402-486-0463. Email: s.kotz@uke.de.
}

Received: January 27, 2013

Accepted: February 26, $2013 \quad$ Online Published: April 25, 2013

DOI : $10.5430 /$ jnep.v3n11p111

URL: http://dx.doi.org/10.5430/jnep.v3n11p111

\begin{abstract}
Objective: This paper will discuss the specific withdrawal symptoms in long term sedation of pediatric intensive care patients, the selection of medication and the special care which these pediatric patients require.

Result: The patients of a pediatric intensive care unit are often postoperative, seriously traumatized, or in a lifethreatening circulatory situation, therefore ventilation, analgesia and sedation, and if necessary muscle relaxation is indicated. This is to reduce stress and to allow invasive procedures.
\end{abstract}

Conclusion: The withdrawal from long term sedation in the context of a pediatric intensive care unit is a big challenge for the nurses, who are the closest to the patients.

\section{Introduction}

Analgesia and sedation medication is available and used for pediatric patients. The choice of drugs is based upon the type of procedure, the anticipated degree of pain, the desired depth of sedation, and the patient's underlying medical condition. Furthermore it is also important to identify the symptoms of opioid and benzodiazepine withdrawal. This paper will discuss the specific symptoms, the selection of medication and the special care which these pediatric patients necessitate.

\section{Definition of terms}

Analgesics are divided into peripherally and centrally-acting medication. Furthermore these drugs are classified by their potency into non opiate (for example non steroid antiphlogistics) and opiate analgesia (morphine, morphine derivative) ${ }^{[3]}$. Some of the important components of analgesia and sedation are anxiolysis, hypnosis, anterograde amnesic effectively and psycho vegetative shielding ${ }^{[6]}$. Long term sedation is not precisely defined in literature. However, it is known that the application of opioids for longer than two to three weeks can cause withdrawal symptoms ${ }^{[8]}$. When administering sedation and opioids to pediatric patients over an extended period of time, one has to check for indication of withdrawal symptoms if the medication is discontinued and administration had exceeded three to four days ${ }^{[9]}$. Physical dependence is defined as a state in which the patient depends on a certain substance or drug (such as opioids or hypnotically drugs) ${ }^{[5]}$. Weaning is the therapy of dependence, usually by reducing the prescribed dose ${ }^{[5]}$. The withdrawal of barbiturate can lead to delirium tremens, similar to alcohol withdrawal ${ }^{[5]}$. Tolerance in the pharmacological way is present if the user has to decrease the interval of application and increase the dose in order to achieve the same desired results ${ }^{[5]}$. 


\section{Effects and side effects in long term conscious sedation}

Since pediatric intensive care patients are usually postoperative, seriously traumatized, or in a life-threatening circulatory situation, ventilation, analgesia and sedation, and if necessary muscle relaxation are required. Undesirable effects and related complication of the used medication can be over dosage, tolerance, delirium and extended artificial ventilation. The benefits of medication are the reduction of stress and the possibility to perform invasive procedures. Nevertheless, after three to four days of constant administration of intravenous sedation and analgesia, the dose has to be adjusted. The adverse effects have to be considered.

\section{Opioids}

The central nervous system with its specific opioid receptors ( $\mu-, \kappa-, \delta$-, and $\sigma$-receptor) is the main area of effect. Through receptor obligation different effects occur, such as analgesia and sedation, breathing depression, tolerance/dependency as well as withdrawal symptoms after the end of opioid administration. Furthermore, opioids cause euphoria and dysphoria, hallucination and the loss of spatiotemporal orientation. Other effects are bradycardia and tachycardia, hypothermia and hyperthermia as well as miosis ${ }^{[8]}$. A respiratory depression can also be observed. Gastrointestinal symptoms are slower forward peristalsis and constipation as well as nausea and vomiting. Frequent diarrhea is an additional burden for the patient, even after extubation. Administration of neuroleptica, benzodiazepine, and clonidine produce increases and prolonged effects of opioids. Coma, miosis and respiratory depression are signals for opioid intoxication ${ }^{[7]}$. The opioid overdose is characterized by a very low breathing frequency with deep breathing and command breathing, which results in a risk of breathing depression with a consecutive circulation failure. Naloxon as the antidote is to be used in the case of an opioid overdose.

\section{Benzodiazepine}

Benzodiazepines cause inhibitory effects in the central nervous system via special benzodiazepine receptors within the GABA-receptor complex. These may be anxiolytic, amnesic, sedative, muscle relaxative, hypnotic and antiepileptic. Benzodiazepines however do not have an analgetic effect. This is achieved by combination with opioids ${ }^{[8]}$. Side effects of benzodiazepine are amongst others tolerance, paradox reactions and the withdrawal syndrome. Furthermore, the ceiling effect has to be considered. In this case saturation of the specific receptors leads to a situation in which even higher levels of benzodiazepine cannot induce a deeper sedation anymore ${ }^{[7]}$. The antagonist for this group of drugs is flumazenil.

\section{Symptoms with reduction of long term sedation}

It can be very difficult to distinguish if a patient experiences pain or is merely agitated. First and foremost, analgesic aspects have to be considered and in a second step, sedation may be adjusted as required ${ }^{[4]}$. In order to provide optimized treatment for patients with withdrawal symptoms it is particularly crucial that the nurse in charge is familiar with the side effects of the therapy and the administered medication. These have to be diagnosed and handled appropriately. Symptoms are as follows.

\section{Symptoms of opioid withdrawal}

The withdrawal from opioid is characterized by agitation, tremor, exudation, vomiting and diarrhea. Some of these symptoms can also occur as side effects of opioid therapy. Only qualified monitoring of the patient allows a distinction between side effects and withdrawal symptoms ${ }^{[1]}$.

\section{Symptoms of benzodiazepine withdrawal}

With the reduction or abrupt discontinuation of the long term sedation with benzodiazepines different symptoms may occur. Due to the over stimulation of the central nervous system the patients frequently show tremor, muscle spasm, dysphoria and restlessness. Dysfunction of the autonomous nervous system may cause exudation, tachycardia and 
yawning. As with the opioid withdrawal the gastrointestinal system is affected and the patient is refusing food and lacks appetite ${ }^{[1]}$. Furthermore anxiety attacks can occur and the risk of seizures is increased.

WithdRAWAL ASSESSMENT TOOL VERSION 1 (WAT - 1)

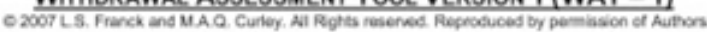

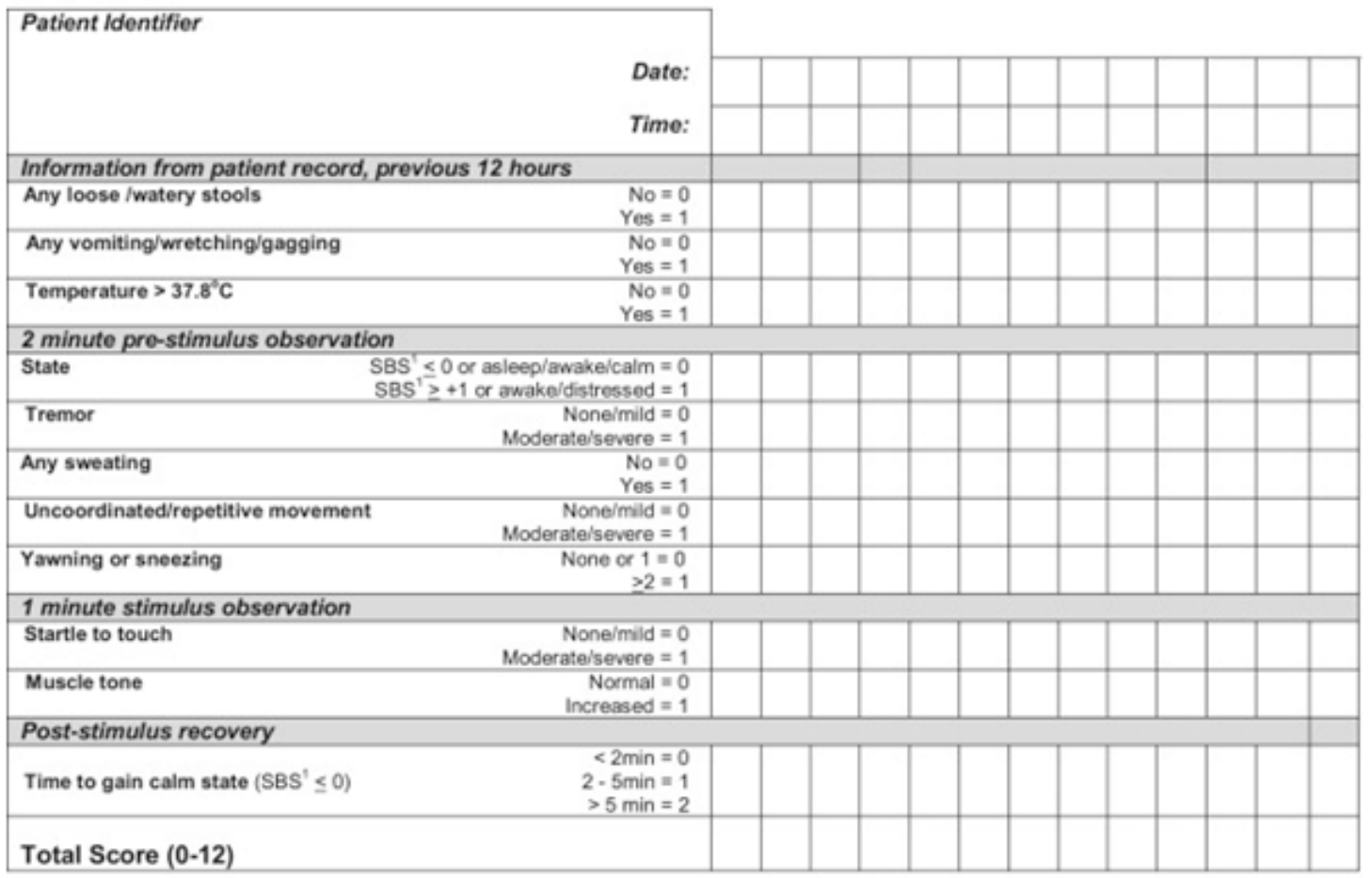

WITHDRAWAL ASSESSMENT TOOL (WAT - 1) INSTRUCTIONS

- Start WAT-1 scoring from the first day of weaning in patients who have received opioids +/or benzodiazepines by infusion or regular dosing for prolonged periods (e.g., > 5 days). Continue wice daily scoring until 72 hours after the last dose.

- The Withdrawal Assessment Tool (WAT-1) should be completed along with the SBS' at least once per 12 hour shift (e.g-, at 08:00 and 20:00 \pm 2 hours). The progressive stimulus used in the SBS' assessment provides a standard stimulus for observing signs of withdrawal.

Obtain information from patient record (this can be done before or after the stimulus):

$\checkmark$ Loose/watery stools: Score 1 if any loose or watery stools were documented in the past 12 hours; score 0 if none were noted.

$\checkmark$ Vomiting/wretching/gagging: Score 1 if any vomiting or spontanecus wretching $o r$ gagging were documented in the past 12 hours; score 0 if none were noted

$\checkmark$ Temperature $>37.8^{\circ} \mathrm{C}$ : Score 1 if the modal (most frequently occurring) temperature documented was greater than $37.8^{\circ} \mathrm{C}$ in the past 12 hours; score 0 if this was not the case.

2 minute pre-stimulus observation:

$\checkmark$ State: Score 1 if awake and distress (SBS': $z+1$ ) observed during the 2 minutes prior to the stimulus; score 0 if asleep or awake and calm/cooperative (SBS' $\leq 0)$.

$\checkmark$ Tremor: Score 1 if moderate to severe tremor observed during the 2 minutes prior to the stimulus; score 0 if no tremor (or only minor, intermittent tremor).

$\checkmark$ Sweating: Score 1 if any sweating during the 2 minutes prior to the stimulus; score 0 if no sweating noted.

$\checkmark$ Uncoordinated/repetitive movements: Score 1 if moderate to severe uncoordinated or repetitive movements such as head turning, leg or arm flailing or torso arching observed during the 2 minutes prior to the stimulus; score 0 if no (or only mild) uncoordinated or repetitive movements. $\checkmark$ Yawning or sneezing > 1: Score 1 if more than 1 yawn or sneeze observed during the 2 minutes prior to the stimulus; score 0 if 0 to 1 yawn or sneeze.

1 minute stimulus observation:

$\checkmark$ Startle to touch: Score 1 if moderate to severe startle occurs when touched during the stimulus; score 0 if none (or mild).

Muscle tone: Score 1 if tone increased during the stimulus; score 0 if normal.

Post-stimulus recovery:

Time to gain calm state (SBS' $\leq 0$ ): Sccre 2 if it takes greater than 5 minutes following stimulus; score 1 if achieved within 2 to 5 minutes; score 0 if achieved in less than 2 minutes.

Sum the 11 numbers in the column for the total WAT-1 score (0-12).



Figure Legend: Withdrawal Assessment Tool (WAT-1) and instructions. 'Reprinted with permission Pediatric Critical Care Medicine 2008;9(6):577.

Figure. Withdrawal Assessment Tool Version 1 (WAT-1) 


\section{Possibilities of withdrawal}

Only once a pediatric patient is in stable condition, withdrawal from long term sedation can be launched. For this it is important that the patient is integrated in the withdrawal efforts. The highest priority should be given to analgesia and anxiety treatment ${ }^{[7]}$.

\section{Medication procedure}

As described in literature, a combination of the benzodiazepine midazolam and the opioid fentanyl is being used in long term sedation. For a deeper sedation, e.g. for an asthmatic patient, ketanest may be administered additionally. Clonidine, an alpha-2 adrenergic agonist, is used to counter withdrawal symptoms. It also allows for reduction of sedatives and analgesics doses ${ }^{[2]}$. Furthermore the autonomous nervous system is slowed down and the effect of benzodiazepine is increased. Side effects such as low blood pressure, bradycardia and deeper sedation can be observed during clonidine administration ${ }^{[7]}$. If tolerance to long term sedation occurs lorazepam may be used for anxiolytic therapy as required ${ }^{[9]}$. For infants, satisfactory results have been obtained using chloral hydrate and/or phenobarbital as an additional medication $^{[2]}$.

\section{Pediatric care}

The pediatric care of a patient with withdrawal symptoms requires a special attention. It is necessary to involve the respective attachment figure in the process and tailor the professional care to the biological rhythm of the patient. The main challenges are insomnia as well as symptoms of fear, agitation and disorientation. The environment should be quiet and the patient should be protected from stress as far as practical ${ }^{[1]}$. Within the first days of withdrawal, diarrhea, fever and heavy sweating have to be handled. In order to categorize the withdrawal symptoms a scale has been developed in 2008 in the USA (The Withdrawal Assessment Tool - Version 1). This scale simplifies the withdrawal process and monitors the opioid and benzodiazepine withdrawal symptoms in pediatric patients significantly. The presence of 19 withdrawal symptoms and the patients overall withdrawal intensity using a numeric rating scale (NRS) where 0 indicated no withdrawal and 10 indicated worst possible withdrawal. Analyzing each symptom in relation to withdrawal intensity ratings, adjusted for site, subject and age group took the result for an 11-item (12-point) scale. Construct validity was supported by significant differences in drug exposure, length of treatment and weaning from sedation, length of mechanical ventilation and intensive care unit stay for patients with WAT- 1 scores $\geq 3$ compared to those with lower scores ${ }^{[3]}$. An instruction to use and The Withdrawal Assessment Toll-Version 1 is shown in this paper ${ }^{[10]}$. The presence of an attachment figure is beneficial for the patient ${ }^{[1]}$. The parents should, if possible, be included in the care of their child, also under long term sedation. They can be informed about effects and side effects of sedation and analgesia. This should improve the parent's ability to support their child during the challenging phase of withdrawal. They may read a book to their child, and, after extubation, also play or draw with them. However, care has to be taken as to sufficient sleep and rest for the patient. Adequately informed parents and an appropriate regime of withdrawal provide comfort for the parents and their child under these stressful circumstances ${ }^{[2]}$.

\section{Conclusion}

The withdrawal from long term sedation in pediatric intensive care represents a big challenge for the concerned nurses. Without interfering with the intensive care therapy, the sedation aims to reduce pain and fear as far as practicable. An intense observation of the patient by the nurse is significant for a successful withdrawal and may be supported by involving an attachment figure (e.g. parent).

\section{References}

[1] Arx-Strässler, F. von. Das zitternde Erwachen- Entzugssymptomatik nach Schmerztherapie. In: Kinderkrankenschwester, Nr. 6, 30. Jg/. 2011: 240-241. 
[2] AWMF: online - S3 - Leitlinie: Analgesie, Sedierung und Delirmanagement in der Intensivmedizin. Download from 07.11.2010. p. 62, p. 74. Available from: http://www.arztbibliothek.de/mdb/downloads/intensivmedizin/dgai/analgesie-sedierung-delirmanagement-mr.pdf.

[3] Franck, L. S.; et al. The Withdrawal Assessment Tool - Version 1 (WAT-1). NIH Public Access Author Manuscript, London. 2008: $1-16$.

[4] Glöckler, M.: Praktische Aspekte der Analgosedierung. Download from 08.11.2010. p.9, p.15.

[5] http://www.akademie.ukerlangen.de/e1852/e1611/e2729/inhalt2768/4_OADr.Gloeckler_Analgosedierung.pdf

[6] HILDEBRANDT, H. Pschyrembel Klinisches Wörterbuch. 258. Auflage. Berlin, New York: Walter de Gruyter. 1998: 3, 328, 434, 1579.

[7] KRETZ, F.-J.; et al. Anästhesie und Intensivmedizin bei Kindern. 2. Auflage. Stuttgart, Erlangen: Thieme. $2007: 356$.

[8] LARSEN, R. Anästhesie und Intensivmedizin für Schwestern und Pfleger. 5. Auflage. Berlin, Heidelberg: Springer. 1999: 164, 759-766.

[9] Latsch, L.; et al. Anästhesie Intensivmedizin Intensivpflege. 2. Auflage. Frankfurt a. M./München: Uraban\&Fischer. 2004: 198, $261,582$.

[10] Schmidtke Dr., S. SOP Analgosedierung auf der Kinderintensivstation UKE. 2.3.35 Version 02. 2010: 1-4, 2-3. 